\title{
PHOTOPHYSICAL PROPERTIES AND ENERGY TRANSFER DYE LASER CHARACTERISTICS OF 7-DIETHYLAMINO-3-HETEROARYL COUMARIN IN SOLUTION
}

\author{
B. BANGAR RAJU ${ }^{1}$ \\ Institute fur Physikalische Chemie, Universität Wien, A 1090, \\ Währingerstrasse 42, Vienna, Austria \\ T. S. VARADARAJAN \\ Physics Section, U.D.C.T., Matunga, Bombay-400 019, India
}

(Received 13 September 1994)

\begin{abstract}
Photophysical properties and Energy transfer Dye Laser (ETDL) characteristics of a new dye: 7-DiethylAmino Coumarin with a Heteroaryl substitution in the 3-position (DACH), are presented. The suitability of $\mathrm{DACH}$ as a laser dye has been verified on the basis of the absorption and fluorescence characteristics in aprotic solvents. The dye laser characteristics of the dye pumped by the available nitrogen laser have been successfully investigated in dimethylsulfoxide by energy transfer dye laser mechanism, using coumarin 1 as a donor dye. DACH has been found to lase in the blue-green region of the visible spectrum. The experimental and theoretical values of energy transfer rate constant $\left(\mathrm{k}_{\mathrm{ET}}\right)$ and the critical transfer distance $\left(\mathrm{R}_{0}\right)$ have been calculated in two different solvents to determine the nature of energy transfer. The results indicate the dominance of resonance transfer in this ETDL.
\end{abstract}

KEY WORDS: Aminocoumarins, Heteroaryl coumarin, Fluorescence quantum efficiency, Energy transfer dye laser, Tunability, Resonance transfer.

\section{INTRODUCTION}

Coumarins and rhodamines form an important class of laser dyes. The coumarin class of laser dyes have a strong electron donating substituent (either hydroxy,$\mathrm{OH}$, or an amino,- $\mathrm{NR}_{2}$ ) in the 7-position. ${ }^{1}$ Coumarin 1(7-diethylamino-4-methyl coumarin) was the first coumarin derivative from which Sorokin and Lankard observed laser action. ${ }^{2}$ Since then about 100 coumarin laser dyes have come to be known. ${ }^{3}$ The 7 -hydroxy coumarins and 7 -aminocoumarins with various electron withdrawing substituents in 3-, 4- and 6-positions provide a large number of laser dyes of choice in the blue-green region. ${ }^{4-8}$

\footnotetext{
${ }^{1}$ Author for Correspondence
} 
Nevertheless, aminocoumarin derivatives have been found to have reduced fluorescence yield in environments of high polarity. This is attributed ${ }^{9}$ to the nonradiative deactivation of these derivatives due to the formation of the Twisted Intramolecular Charge Transfer (TICT) state from the singlet excited state, $S_{1}$. The population of the TICT state depends on the electron donor-acceptor capacities of the involved partners and on the solvent polarity which stabilizes the highly polar TICT state. ${ }^{10}$ The concept of the TICT state and the role of solvent in the twisting of the bichromophoric molecule in the excited state resulting in such a state was first made prominent by Grabowski et al. ${ }^{11}$ to explain the dual fluorescence of $\mathrm{N}, \mathrm{N}$ dimethylaminobenzonitrile in polar solvents.

For designing efficiently fluoresceing systems, for example laser dyes, it is very important ${ }^{12}$ to control the formation of the TICT state because this often acts as intramolecular quencher. One of the possible approaches to achieve this is to reduce the strength of the acceptor substituent ${ }^{12}$ which makes the energy of the TICT state higher than that of the ICT state, thus preventing the population of the former state.

In the course of our on going research to find new laser dyes, we envisaged the concept of varying the strength of the substituent in the 3-position within the class of 7-aminocoumarins exhibiting good laser characteristics and study its effect on the photophysical properties and lasing characteristics in various solvents. On the basis of this several new 7-diethylamino coumarins with varying electron acceptor substituents in 3-position have been synthesised ${ }^{13}$ and two new dyes lasing in the blue-green region have been successfully identified. ${ }^{14,15}$

In the work presented here we have investigated the photophysical properties and the-available nitrogen laser pumped-energy transfer dye laser characteristics (ETDL) of a new dye: 7-DiethylAmino Coumarin with a Heteroaryl substitution in the 3position (DACH) in solution. The effect of the concentration of the components of the dye mixture on these characteristics is also studied and discussed. Fluorescence energy transfer characteristics are determined, both experimentally and theoretically, to ascertain the nature of the transfer mechanism involved in this dye mixture.

\section{EXPERIMENTAL}

The dye DACH has been synthesized ${ }^{13}$ and supplied in the pure form by Seshadri and Shenoy of the Dyes Research Laboratory of U.D.C.T., Bombay, India. The solvents used viz chloroform $\left(\mathrm{CHCl}_{3}\right)$, ethanol, dioxane, DiMethylFormamide (DMF) and DiMethylSulfOxide(DMSO) were all of spectroscopic grade from S. D. Fine Chemicals, India. The choice of solvents is based on the requirement of solubility in the range of $10^{-4}-10^{-2} \mathrm{~mol} / 1$ to achieve laser action. Rhodamine $B$ was from B.D.H., U.K. The purity of the dyes and solvents has been verified by spectroscopic and chromatographic (tlc) techniques prior to use.

Absorption spectra of the dyes and their mixtures were recorded on Milton Roy spectronic $1201 \mathrm{UV}$-visible absorption spectrophotometer. The fluorescence spectra were recorded on Aminco Bowman spectrofluorophotometer using front-surface excitation geometry. A Riken Denshi F-3G X-Y/t recorder was used to record fluorescence spectra. Necessary corrections were applied for grating monochromator 
and photomultiplier tube (PMT) (IP28) sensitivities. Rhodamine B (in ethanol) was used as a standard to determine the fluorescence quantum efficiency. ${ }^{16}$ Fluorescence quantum efficiencies were calculated by using a quadratic correction for the refractive index of the solvent and a correction for the percent of light absorbed by the samples. For these measurements dilute solutions of DACH and Rhodamine $\mathrm{B}$ (maximum $\mathrm{OD}<0.2$ ) were employed with excitation at the absorption maximum. Laser action of the dye mixture was tested using a pulsed nitrogen laser (Jobin Yvon LA 04, peak power $400 \mathrm{~kW}$ with a pulse width of $8 \mathrm{~ns}$ and operated at a repitition rate of 15-20 pps) with a transverse dye laser oscillator cavity. The dye laser output was detected through a monochromator (Jobin Yvon model H200V), PMT(IP28) and a X-Y/t recorder (Riken Denshi F-3G). A corrected dye laser output was obtained after performing the necessary corrections for the sensitivities of the grating/monochromator and PMT.

\section{RESULTS AND DISCUSSION}

\subsection{Photophysical Properties}

The absorption and fluorescence characteristics of DACH in dioxane and DMSO are shown in Figure 1 and the photophysical characteristics are listed in detail in Table 1.

The observations are as follows. There is a general hypsochromic shift of the absorption and fluorescence spectra with increase in dielectric constant of the

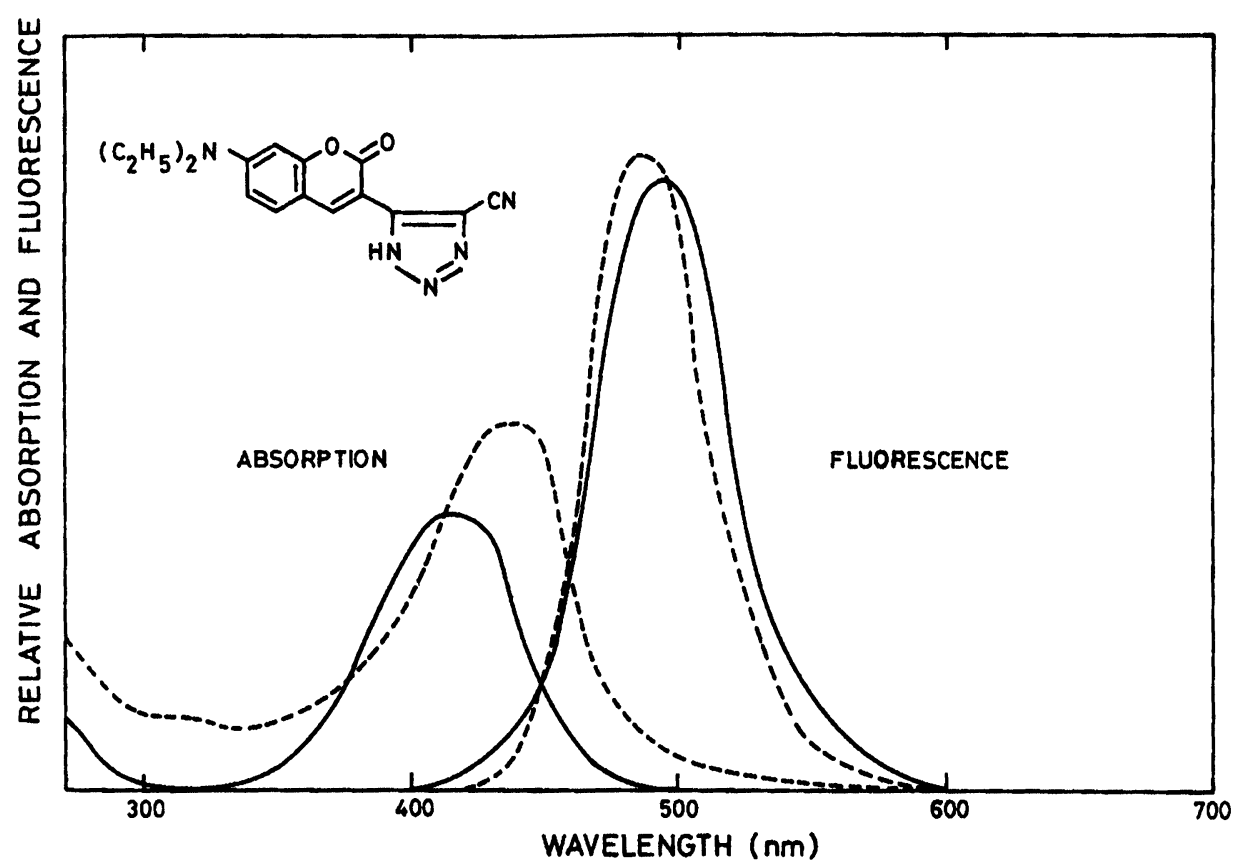

Figure 1 Absorption and fluorescence spectra of DACH in DMSO (- - ) and DIOXANE(----). 
solvent (solvent polarity), with the exception in DMF. The absorption spectrum is more sensitive to the changes in solvent polarity than the emission spectrum. Thus the maximum wavelength of absorption shifts by nearly $50 \mathrm{~nm}$ when the solvent is changed from $\mathrm{CHCl}_{3}\left(\lambda_{\mathrm{ab}}=453 \mathrm{~nm}\right)$ to $\operatorname{DMSO}\left(\lambda_{\mathrm{ab}}=401 \mathrm{~nm}\right)$. The emission maximum also shifts to higher energies in solvents of greater polarity, however, the magnitude of the shift is not as large as that observed in the absorption spectrum. The hypsochromic shift of the absorption and fluorescence spectrum in polar solvents could, although, mean that the dye is less polar in the excited state than in the ground state, this cannot explain the large Stokes shift of the dye in solvents like DMF and DMSO, which indicates an increase in the dipole moment upon excitation. These anomalous spectral characteristics could be rather due to specific solutesolvent interactions resulting from the charge transfer reactions. ${ }^{15}$

The fluorescence quantum efficiency $\phi_{f \mid}$, is reasonably high, between 0.50 and 0.70 , and the fluorescence lifetime, $\tau_{f f}$, is of the order suitable ${ }^{1}$ for a good laser dye (around 3ns), in the range of solvents studied. Both these values are higher in highly polar solvents.

The observed absorption and fluorescence properties of DACH can be understood in terms of the heteroaryl substituent at the 3-position. Heteroaryl substituents at the 3-position are shown ${ }^{17}$ to increase the fluorescence property of aminocoumarin moiety. Dyes having this type of conjugation are closely related to the styryl dyes in that they may be considered as possessing a ring type styryl structure $^{18}$ in the manner shown below:



DACH has an extended conjugation and thus displays an unconstrained, but not freely rotating substituent at the 3-position. The electron withdrawing strength is thus reduced due to the extended delocalization. The interaction between the donor and the acceptor substituents is compromised by the additional $\pi$-conjugation. Thus, due to the rotation of the unconstrained diethylamino group, the formation of the TICT state is although still possible, the energy of this state is perhaps unfavourable compared with that of the ICT state. Hence the twisting relaxational motion

Table 1 Absorption and fluorescence characteristics of DACH in various solvents

\begin{tabular}{lccccc}
\hline Solvent & $\begin{array}{c}\lambda_{\mathrm{a}} \\
(\mathrm{nm})\end{array}$ & $\begin{array}{c}\varepsilon_{\mathrm{a}} * 10^{-4} \\
(1 / \mathrm{mol}-\mathrm{cm}))\end{array}$ & $\begin{array}{c}\lambda_{\mathrm{f} 1} \\
(\mathrm{~nm})\end{array}$ & $\phi_{\mathrm{f1}}$ & $\begin{array}{c}\tau_{\mathrm{f1}} \\
(\mathrm{ns})\end{array}$ \\
\hline CHCl $_{3}$ & 453 & 6.65 & 490 & 0.61 & 2.78 \\
DIOXANE & 435 & 4.51 & 486 & 0.48 & 2.76 \\
DMF & 401 & 2.15 & 480 & 0.65 & 2.85 \\
DMSO & 404 & 2.98 & 484 & 0.69 & 2.87 \\
\hline
\end{tabular}


may not be taking place. This could be reason for the sustained fluorescence of DACH in solvents of even higher dielectric constant.

When $\phi_{\mathrm{fl}}$ and $\tau_{\mathrm{fl}}$ are both available, the solvent dependency can be resolved in terms of the influence of the radiative $\left(\mathrm{k}_{\mathrm{r}}\right)$ and the nonradiative $\left(\mathrm{k}_{\mathrm{nr}}\right)$ rate constants, ${ }^{10}$ given by:

$$
\mathrm{k}_{\mathrm{r}}=\phi_{\mathrm{f} 1} / \tau_{\mathrm{f} 1}
$$

and

$$
\mathrm{k}_{\mathrm{nr}}=\left(1-\phi_{\mathrm{fl}}\right) / \tau_{\mathrm{fl}}
$$

These values, listed in Table 2, show a greater influence of the solvent on $\mathrm{k}_{\mathrm{nr}}$. The value of $\mathrm{k}_{\mathrm{nr}}$ is comparable with that obtained for other coumarin laser dyes. ${ }^{8,19}$

An important indication for the quality of laser dyes is the cross section of absorption $\left(\sigma_{\mathrm{a}}(\lambda)\right)$ and fluorescence $\left(\sigma_{\mathrm{e}}(\lambda)\right) .{ }^{1} \mathrm{~A}$ higher value of the absorption cross section at the wavelength of the pump laser is especially desirable for a good laser dye. ${ }^{20}$

The absorption cross section is given by ${ }^{1}$ :

$$
\sigma_{\mathrm{a}}(\lambda)=0.385 * 10^{-20} \varepsilon_{\max }
$$

where, $\varepsilon_{\max }$ is the molar extinction co-efficient of the longest wavelength maximum of absorption.

The fluorescence cross section is given by ${ }^{21}$ :

$$
\sigma_{\mathrm{e}}(\lambda)=\lambda_{\mathrm{fl}}^{4} \mathrm{~F}(\lambda) \phi_{\mathrm{fl}} / 8 \pi \mathrm{cn}^{2} \tau_{\mathrm{fl}}
$$

where, $F(\lambda)$ is the normalized fluorescence line shape function, such that $\int F(\lambda)$ $\mathrm{d} \lambda=1, \mathrm{n}$ is the refractive index of the solvent and $\mathrm{c}$ is the velocity of light.

The absorption cross section of DACH at the wavelength of the pump laser is found to be low $\left(<3.5 * 10^{-17} \mathrm{~cm}^{-1}\right)$, due to which efforts to pump this dye, directly above threshold, using the available nitrogen laser have been unsuccessful.

We have attempted to obtain laser action from this dye by means of indirect excitation by energy transfer mechanism. For this purpose we used coumarin 1 as the donor dye because it has a short fluorescence lifetime, ${ }^{5}$ it lases on direct excitation by nitrogen laser ${ }^{1}$ and because the fluorescence spectrum of coumarin 1 overlaps the absorption spectrum of DACH (Figure 2) indicating suitability for energy transfer.

We present below, the results of the Energy Transfer Dye Laser (ETDL) characteristics of the above dye laser system in DMSO.

Table 2 Photophysical properties of DACH in in various solvents

\begin{tabular}{lcccc}
\hline Solvent & $\begin{array}{c}k_{\mathrm{r}} * 10^{-8} \\
\left(S^{-1}\right)\end{array}$ & $\begin{array}{c}k_{\mathrm{nr}} * 10^{-8} \\
\left(S^{-1}\right)\end{array}$ & $\begin{array}{c}\sigma_{\mathrm{a}}(\lambda) * 10^{-17} \\
\left(\mathrm{~cm}^{2}\right)\end{array}$ & $\begin{array}{c}\sigma_{\mathrm{e}}(\lambda) * 10^{-17} \\
\left(\mathrm{~cm}^{2}\right)\end{array}$ \\
\hline CHCl $_{3}$ & 2.19 & 1.40 & 25.60 & 11.42 \\
DIOXANE & 1.74 & 1.88 & 17.36 & 4.53 \\
DMF & 2.28 & 1.23 & 8.23 & 5.07 \\
DMSO & 2.40 & 1.08 & 11.57 & 5.37 \\
\hline
\end{tabular}




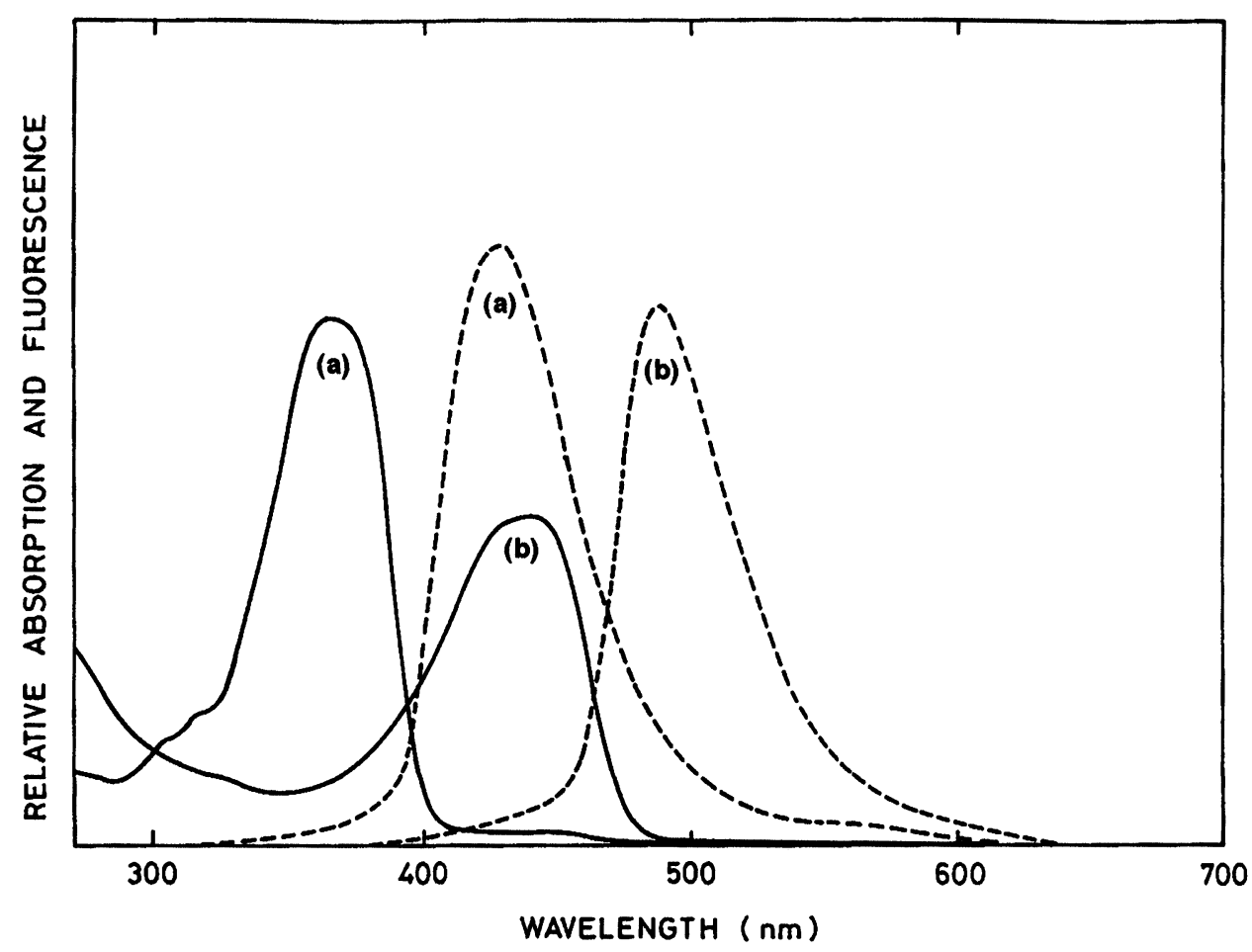

Figure 2 Absorption (_- ) and fluorescence (-...-) spectra of (a) Coumarin 1 and (b) DACH in DIOXANE.

\subsection{Energy Transfer Dye Laser Characteristics of $D A C H$}

Figure 3 shows the effect of the concentrations of the components:donor and acceptor, on the laser characteristics of the later. The concentration of donor and acceptor has been optimized for maximum energy transfer conversion efficiency.

The ETDL characteristics were studied under the following conditions:

(1) Maximum Laser Intensity $\left(I_{L}\right)$ and the wavelength at which it occurs $\left(\lambda_{L}\right)$ were determined for different concentrations of donor, from $1^{*} 10^{-3} \mathrm{~m} / 1$ to $1 * 10^{-2} \mathrm{~m} / 1$, at a fixed acceptor concentration of $2.5 \times 10^{-3} \mathrm{~m} / 1$.

(2) $I_{L}$ and $\lambda_{L}$ were measured for different concentrations of acceptor, from $1 * 10^{-3} \mathrm{~m} / 1$ to $1 * 10^{-2} \mathrm{~m} / 1$, keeping the donor concentration fixed at $1.5 \times 10^{-3} \mathrm{~m} / 1$.

The observations are presented in Figures 3(A) and 3(B), respectively.

A first look at Figures 3(A) and 3(B) shows that considerably high concentration of the components was required to achieve lasing action from the sensitized acceptor. This could be rationalized as due to the smaller value of the cross section of fluorescence emission of DACH, which is an order of magnitude less than that of the standard laser dyes, rhodamine $6 \mathrm{G}$ and rhodamine $\mathrm{B} .{ }^{22}$ 


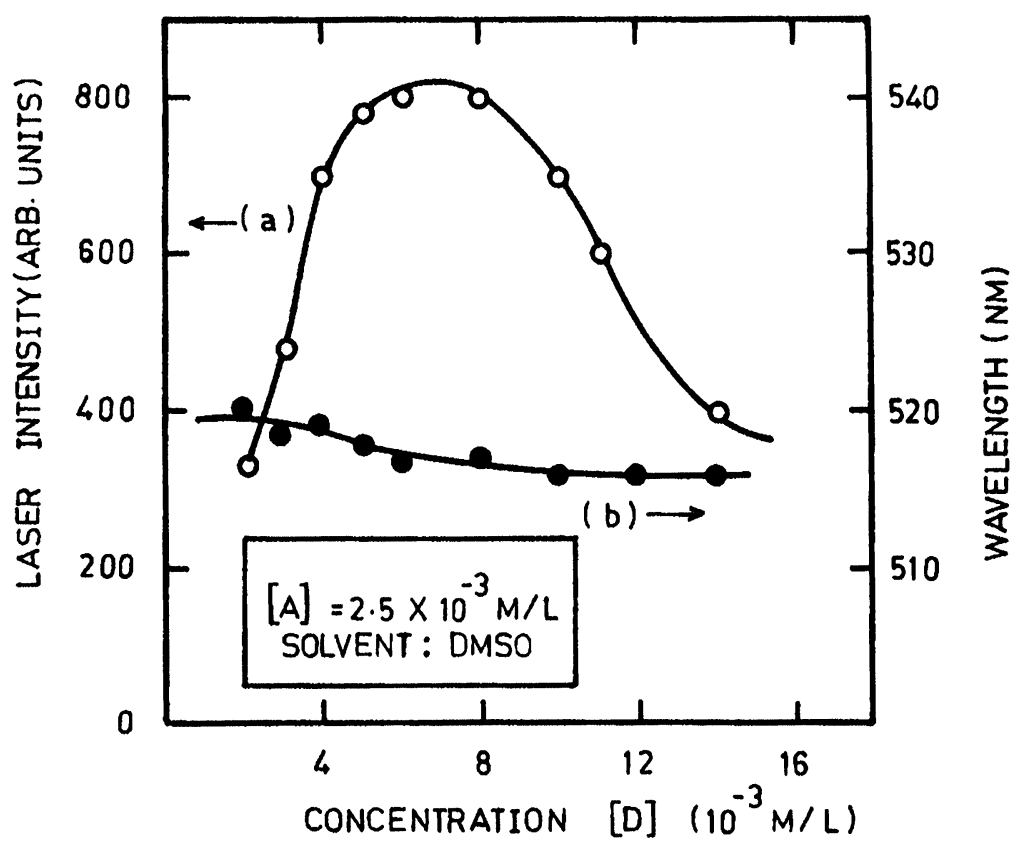

Figure 3(A) (a) Laser intensity and (b) wavelength of the dye mixture as a function of concentration of the donor.

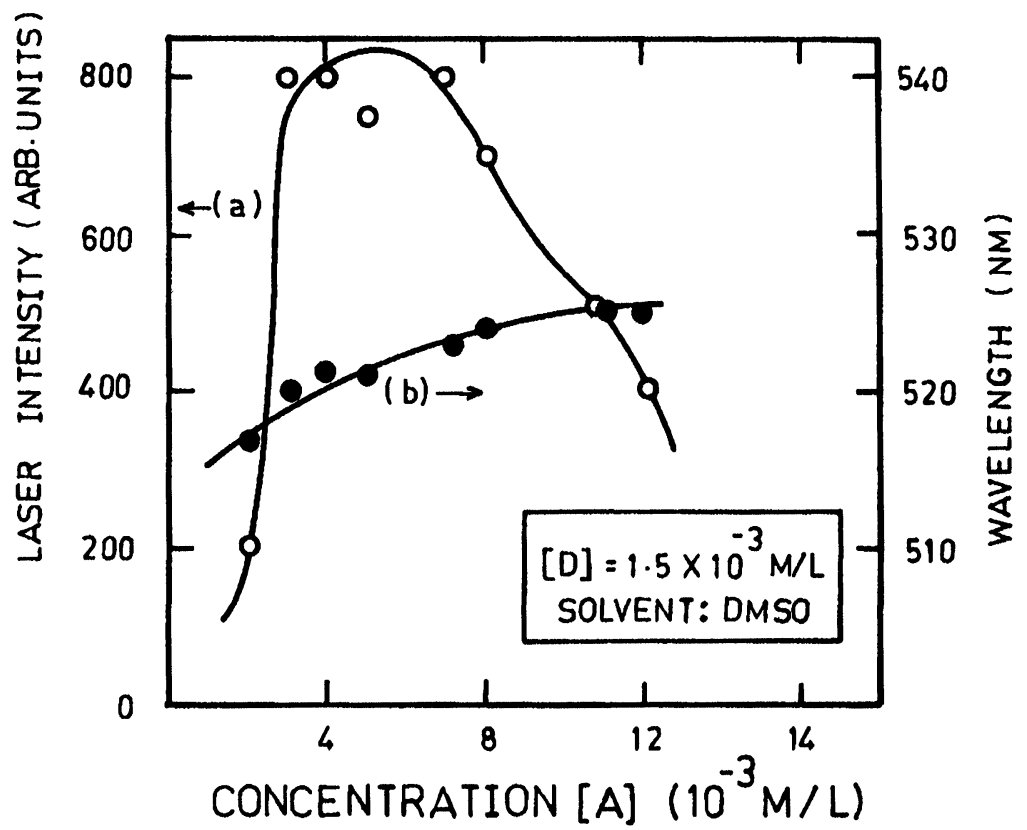

Figure 3(B) (b) Laser intensity and (b) wavelength of the dye mixture as a function of concentration of the acceptor. 
Since fairly high concentrations of donor and acceptor were required to obtain the laser action, this is reflected in the noticeable red shift of the peak laser emission (between 515 and $525 \mathrm{~nm}$ ) from the fluorescence maximum $(484 \mathrm{~nm})$ of the sensitized acceptor, indicating a concentration tuning effect. ${ }^{14}$

Figure 3(A) demonstrates that for low concentrations of the donor, the laser is below the threshold and hence no lasing action takes place. Increasing the concentration of the donor beyond $2 * 10^{-3} \mathrm{~m} / 1$, enables laser action showing clear energy transfer. The wavelength of the ETDL is around $515 \mathrm{~nm}$ and is part of the fluorescence spectrum of the acceptor. This establishes the fact that lasing takes place from the acceptor only. Thus most of the excitation energy absorbed by the donor is efficiently transfered to the acceptor as a useful pump power.

The intensity of laser emission is found to gradually increase with increase in donor concentration and reaches a maximum for a certain value of donor concentration. A further increase in the donor concentration decreased the laser output due to self absorption or formation of non-fluorescent dimers (concentration quenching) of the donor molecules, which gradually increase at such high concentrations, leading to a decrease of the efficiency of energy transfer. Another noticeable observation in these characteristics is the small blue shift (around $5 \mathrm{~nm}$ ) in the peak lasing wavelength of the acceptor with increase in donor concentration (Figure 3(A)). Such concentration dependent blue shifts have also been reported to occur in widely used laser dyes such as coumarin 1 and rhodamine B, etc. ${ }^{1}$

Figure 3(B) demonstrates the changes in the laser output and the lasing wavelength of the dye mixture for different concentrations of the sensitized acceptor, at a fixed concentration of the donor. It can be observed here that at low concentrations of acceptor, the efficiency of energy transfer is low and insufficient to produce lasing action from the dye. The acceptor here acts as a loss filter for the donor. On increasing the concentration of the acceptor, this effect is overcome and the threshold is reached and the dye lases.

On further increasing the acceptor concentration, the laser intensity increases, reaches a maximum value and then begins to decrease. This decrease may be due to the higher ground state absorption by the surrounding acceptor molecules or due to the formation of non-fluorescent dimers among the acceptor molecules which is highly probable at such concentrations. This behaviour is observed in other efficient ETDL systems as well. ${ }^{23-25}$

It can also be observed from Figure 3(B) that the lasing wavelength changes with the increase in acceptor concentration establishing the fact that the lasing takes place, again, from the acceptor only. As the concentration of the acceptor is increased its stimulated emission peak is bathochromically shifted, from $513 \mathrm{~nm}$ to $525 \mathrm{~nm}$, due to increased self-absorption at shorter wavelengths.

\subsection{Fluorescence Energy Transfer Characteristics}

In addition to the above ETDL studies, fluorescence energy transfer characteristics of the dye mixture have been studied, to determine the rate constant for energy transfer $\left(\mathrm{k}_{\mathrm{ET}}\right)$, and also to ascertain the nature of the energy transfer involved in the dye mixture. The plots of the steady-state fluorescence quenching of the donor with 


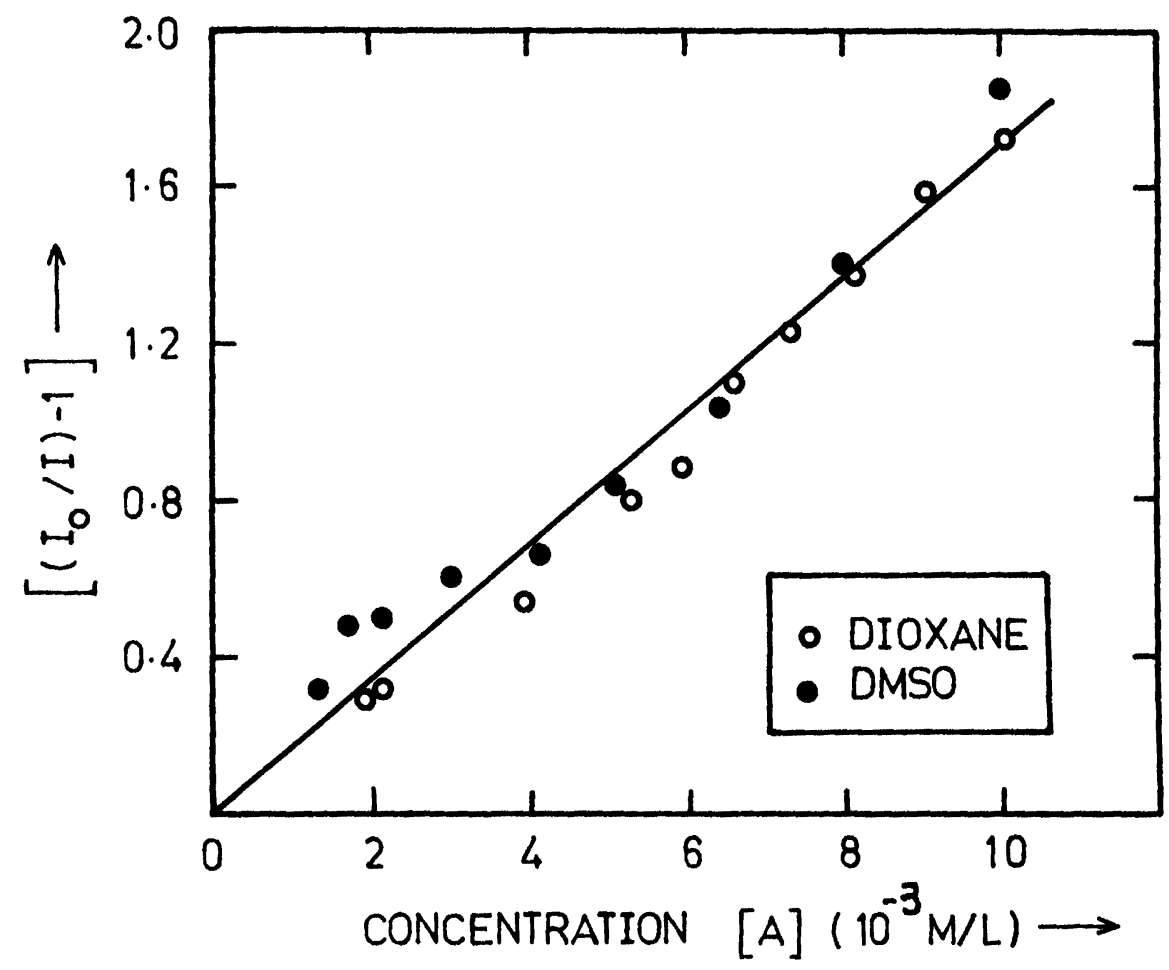

Figure 4 Stern-Volmer plots for fluorescence quenching.

different acceptor concentrations are straight lines (Figure 4), obeying Stern-Volmer relation. ${ }^{26}$ The quenching constants $\left(\mathrm{K}_{\mathrm{sv}}\right)$ are then the slopes of the Stern-Volmer plots for the dye mixtures in various solvents.

The fluorescence energy transfer rate constant $\left(\mathrm{k}_{\mathrm{ET}}\right)$ can then be calculated using the relation ${ }^{27}$ :

$$
\mathrm{k}_{\mathrm{ET}}=\mathrm{K}_{\mathrm{SV}} / \tau_{\mathrm{D}}
$$

where, $\tau_{\mathrm{D}}$ is the fluorescence lifetime of the pure donor in the respective solvents. This value is taken from ref. ${ }^{5}$.

The critical transfer distance, $R_{0}$ (in $\AA$ ), which is the average distance between the donor and the acceptor at which the probability of energy transfer is as high as any other relaxation process is experimentally determined from the Stern-Volmer plots using the relation ${ }^{28}$ :

$$
\mathrm{R}_{0}=7.35 /[\mathrm{A}]_{1 / 2}^{1 / 3}
$$

where, $[\mathrm{A}]_{1 / 2}$ is the concentration of the acceptor required to decrease the fluorescence of the donor by one-half. A summary of these results is presented in table 3 .

The critical transfer distance, $R_{0}$ (in $\AA$ ) which is the average distance between the donor and acceptor at which the probability of energy transfer is as high as 
Table 3 Theoretically calculated and experimentally determined critical transfer distance and rate constants for energy transfer in dioxane and DMSO.

\begin{tabular}{lllll}
\hline Solvent & $\begin{array}{l}R_{0}^{*} \\
(\AA)\end{array}$ & $\begin{array}{l}R_{0}^{* *} \\
(\AA)\end{array}$ & $\begin{array}{l}K_{S V}^{*} 10^{-3 * *} \\
\left(\mathrm{lmol}^{-1}\right)\end{array}$ & $\begin{array}{l}K_{E T^{*}} 10^{-11 * *} \\
\left(\mathrm{lmol}^{-1} \mathrm{~s}^{-1}\right)\end{array}$ \\
\hline Dioxane & 39 & 41 & 1.73 & 6.16 \\
DMSO & 38 & 41 & 1.72 & 5.36 \\
\hline
\end{tabular}

* Calculated using Förster's formula.

** Experimentally determined from stern-volmer plots for bimolecular quenching.

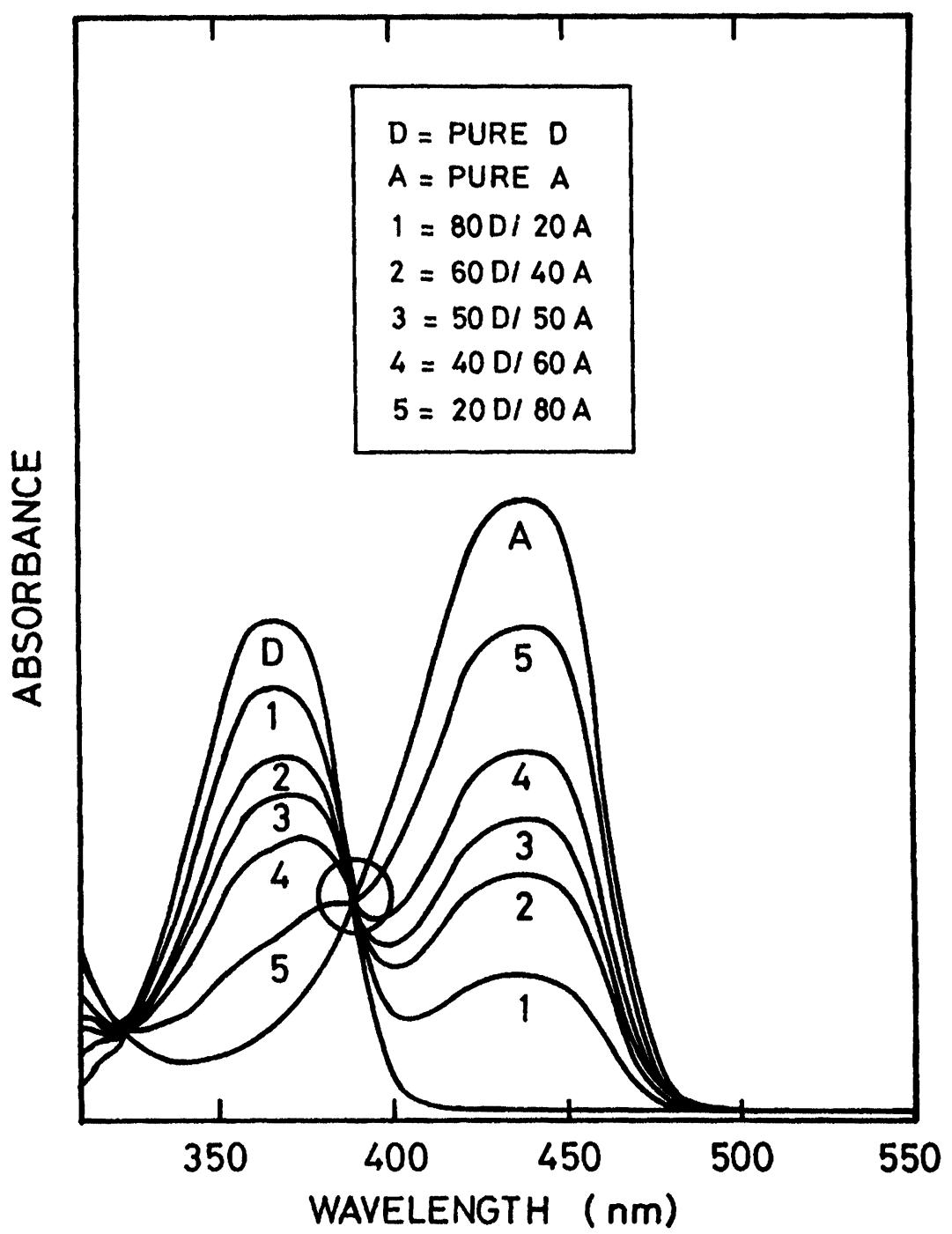

Figure 5 Absorption spectra of coumarin 1 and DACH Mixture in Dioxan. 
any other relaxation mode, has been calculated from the spectral properties of the donor and the acceptor using Förster's relation ${ }^{27}$ of nonradiative energy transfer for long-range dipole-dipole interactions:

$$
\mathrm{R}_{0}^{6}=9000 \ln (10) \mathrm{k}^{2} \phi_{\mathrm{D}}^{\mathrm{o}} \mathrm{J} / 128 \pi^{5} \mathrm{Nn}^{4}
$$

where $\mathrm{k}^{2}$ is the orientation factor assumed to be $2 / 3$ for solution studies, $\phi_{\mathrm{D}}^{\circ}$ is the emission quantum yield of donor in the absence of the acceptor, $n$ is the refractive index of the solvent, $\mathrm{N}$ is the Avagadro's number and $\mathrm{J}$ is the spectral overlap integral.

The value of $\mathrm{R}_{0}$ obtained is $39 \AA$ and $38 \AA$ in dioxane and DMSO respectively, which indicates that significant energy transfer can take place at relatively high donor-acceptor separation distances. These values are also summarised in Table 3.

On the basis of these results and on the following observations, we reject the energy transfer due to Collisional Transfer(CT) and complexing for the present system:

(1) The rate constant for energy transfer obtained here is greater than that obtained in the case of CT, as observed experimentally or calculated from Debye's equation. ${ }^{23-25}$

(2) The absorption spectrum of the donor was found to remain unchanged on addition of acceptor (Figure 5).

(3) No new fluorescence peak was detected in the mixture to indicate any exciplex formation.

The critical transfer distance computed here agrees well with that obtained in energy transfer due to long-range dipole-dipole interactions in other donor-acceptor pairs $^{23-25}$ and is considerably greater than the value for CT. In terms of $k_{E T}$ also the excitation transfer rate constant obtained here is typical of resonance transfer (which is of the order of $10^{9}-10^{10} 1 \mathrm{~mol}^{-1} \mathrm{~s}^{-1}$ ) 23 . The invariance of $R_{0}$ and $\mathrm{k}_{\mathrm{ET}}$ with the solvent is also indicative of the dominance of resonance transfer in the dye mixture under consideration.

The present study thus indicates the dominance of resonance transfer in this ETDL.

\section{CONCLUSIONS}

It can be concluded that by substituting a delocalising substituent at the 3-position of 7-diethylamino coumarin, the rotational deactivation of the molecule, leading to an emission from the TICT state can be overcome, leading to not only a sustained fluorescence, but also lasing action from the dye. Thus DACH lases by energy transfer dye laser mechanism in the blue-green region. Resonance Transfer due to long-range dipole-dipole interactions appears to be the dominant mechanism, although radiative transfer could also be probably contributing to the energy transfer process at the critical transfer distances obtained.

\section{Acknowledgements}

The authors are very thankful to Prof. S. Seshadri and Dr. V. U. Shenoy of the Dyes Research Laboratory of U.D.C.T. for the supply of the dye DACH in the 
pure form. We are thankful to Dr. Tulsi Mukherjee of the Chemistry Division of B. A. R. C. Bombay for helping in determination of the fluorescence lifetimes.

\section{References}

1. K. H. Drexhage, in Dye Lasers: Topics in Applied Physics, Vol. 1, $\left(2^{\text {nd }}\right.$ ed)., F. P. Schäfer (Springer Verlag, New York 1977).

2. M. R. Kagan, G. J. Farmer, B. G. Huth: Laser Focus, 4, 26(1968).

3. M. Maeda: Laser Dyes, (Academic Press, New York 1984).

4. P. S. Song, M. L. Houten, T. A. More, W. C. Herndon: J. Photochem. Photobiol., 14, 521 (1971).

5. G. Chu, F. Yangbo: J. Chem. Soc. Faraday Trans. I, 83, 2533(1987).

6. C. E. Wheelock: J. Amer. Chem. Soc., 81, 1348(1959).

7. P. R. Hammond, A. N. Fletcher, R. A. Henry, R. L. Atkins: Appl. Phys., 8, 311(1975).

8. T. G. Pavlopoulos, J. H. Boyer, I. R. Politzer, Chun N. Lau: Optics Commun., 64, 367(1987).

9. T. L. Arbeloa, F. L. Arbeloa, M. J. Tapia, I. L. Arbeloa: J. Phys. Chem., 97, 4704(1993) (and references therein).

10. G. Jones II, C. Y. Choi, W. R. Jackson, W. R. Bergmark: J. Phys. Chem., 89, 294(1974).

11. Z. R. Grabowski, K. Rotkiewicz, A. Siemainczuk, D. J. Cowley, W. Baumann: Nouveau J. Chim., 8, 443(1979).

12. W. Rettig: Angew. Chem. Int. Ed. Engl., 25, 971 (1986).

13. V. U. Shenoy, Doctoral Dissertation, Bombay University (1990).

14. B. Bangar Raju, T. S. Varadarajan: J. Luminisc., 55, 49 (1993).

15. B. Bangar Raju, T. S. Varadarajan: Appl. Phys. B, 59, 83(1994).

16. I. B. Berlman, Handbook of Fluorescence Spectra of Aromatic Compounds, $\left(2^{\text {nd }}\right.$ ed.), (Academic Press, New York 1971).

17. A. Chandrasekhar, Doctoral Dissertation, Bombay University (1984).

18. P. Moeckeii: Dyes and Pigments, 1, 3(1980).

19. G. Jones II, W. R. Jackson, S. Kanoktanporn, A. M. Halpern: Optics Commun., 33, 315(1980).

20. H. Guesten, M. Rinke, H. O. Writh: J. Appl. Phys., 45B, 279(1988).

21. O. G. Peterson, J. P. Webb, W. C. McColgin, J. H. Eberly: J. Appl. Phys., 42, 1917(1971).

22. L. G. Nair: Progr. Quant. Electr., 7, 153(1982).

23. R. G. Bennet, R. E. Kellog: J. Photochem. Photobiol., 7, 571 (1968).

24. W. Ware: J. Amer. Chem. Soc., 83, 4374(1961).

25. C. Lin, A. Dienes: J. Appl. Phys., 44, 5050(1973).

26. O. Stern and V. Volmer, Physics Z., 20, 183(1919).

27. Th. Förster: Disc. Faraday Soc., 27, 7 (1959).

28. N. J. Turro, in "Modern Molecular Photochemistry" (Benjamin/Cummings Co., California, 1979) Chap. 9. 